\title{
Cardiac Tomography and Cardiac Magnetic Resonance to Predict the Absence of Intracardiac Thrombus in Anticoagulated Patients Undergoing Atrial Fibrillation Ablation.
}

Fatima Zaraket ( $\square$ zarakettt@gmail.com )

Hospital Del Mar

Bas Deva

Hospital Del Mar

Jesus Jimenez

Hospital Del Mar

Benjamin Casteigt

Hospital Del Mar

Begoña Benito

Hospital Del Mar

Julio Martí-Almor

Hospital Del Mar

Javi Conejos

Hospital Del Mar

Diana Mojón

Hospital Del Mar

Helena Tizón-Marcos

Hospital Del Mar

Ermengol Vallès

Hospital Del Mar

\section{Research Article}

Keywords: Atrial fibrillation, intracardiac echography, advanced imaging techniques, atrial fibrillation ablation

Posted Date: August 26th, 2021

DOI: https://doi.org/10.21203/rs.3.rs-775570/v1 
License: (c) (i) This work is licensed under a Creative Commons Attribution 4.0 International License. Read Full License

Version of Record: A version of this preprint was published at Journal of Clinical Medicine on April 8th, 2022. See the published version at https://doi.org/10.3390/jcm11082101. 
Cardiac tomography and cardiac magnetic resonance to predict the absence of intracardiac thrombus in anticoagulated patients undergoing atrial fibrillation ablation

Running title: Advanced cardiac imaging techniques to rule out thrombus in atrial fibrillation

\author{
*Fatima Zaraket ${ }^{1}$, Deva Bas ${ }^{1}$, Jesus Jimenez ${ }^{1}$, Benjamin Casteigt ${ }^{1}$, Begoña \\ Benito ${ }^{1}$, Julio Martí- Almor ${ }^{1}{ }^{2}$, Javi Conejos ${ }^{1}$, Helena Tizón ${ }^{1}$, Diana Mojón ${ }^{1}$, \\ Ermengol Vallès ${ }^{1,2}$
}

${ }^{1}$ Electrophysiology Unit, Cardiology Department, Hospital del Mar.

${ }^{2}$ Universitat Autònoma de Barcelona. Institut Hospital del Mar Investigacions Mèdiques (IMIM). Barcelona

Disclosures: No disclosures Total

word count: 3455

\title{
Address for Correspondence:
}

Fatima Zaraket.

Hospital del Mar. Cardiovascular division.

Electrophysiology Unit. 25-27 Passeig marítim de la

Barceloneta, Barcelona, 08003 Spain. Telephone: 0034

932483118 / Fax: 0034932483371

Email: zarakettt@gmail.com 


\begin{abstract}
Background: Pulmonary veins isolation (PVI) is a standard treatment for recurrent atrial fibrillation (AF). Uninterrupted anticoagulation for minimum 3 weeks before the ablation and exclusion of left atrial (LA) thrombus with transesophageal echography (TEE) immediately before or during the procedure minimize peri-procedural risk. We aimed to demonstrate the utility of cardiac tomography (CT) and cardiac magnetic resonance (CMR) to rule out LA thrombus prior to PVI.
\end{abstract}

Methods: Patients undergoing PVI for recurrent AF were retrospectively evaluated. Only patients that started anticoagulation at least 3 weeks prior to the CT/CMR and subsequently uninterrupted until the ablation procedure were selected. An intracardiac echo (ICE) catheter was used in all patients to evaluate LA thrombus. The results of CT/CMR were compared to ICE imaging.

Results: We included 272 consecutive patients averaging 54.5 years (71\% male; 30\% persistent AF). Average CHA2DS2VASC Score was 0.9 and mean LA diameter was 43 mm, 111 patients on Acenocumarol and 161 on direct oral anticoagulants. Anticoagulation was started $227 \pm 392$ days before the CT/CMR, and 291 $\$ 416$ days before the ablation procedure. CT/CMR diagnosed intracardiac thrombus in 2 cases, both in the LA appendage. A new CT/CMR revealed resolution of thrombus after 6 additional months of uninterrupted anticoagulation. No macroscopic thrombus was observed in any patients with ICE (negative predictive value of $100 \%$; $\mathrm{p}<0.01$ ). 
Conclusions: CT and MRI are excellent surrogates to TEE and ICE to rule out intracardiac thrombus in patients adequately anticoagulated prior AF ablation. This is true even for delayed procedures as long as anticoagulation is uninterrupted.

Key words: Atrial fibrillation, intracardiac echography, advanced imaging techniques, atrial fibrillation ablation.

\section{Introduction}

Left atrium (LA) catheter ablation with pulmonary vein isolation (PVI) is widely employed as treatment for patients with symptomatic, drug refractory atrial fibrillation (AF). Transesophageal echocardiography (TEE) is considered the gold standard for detecting LA thrombus prior to catheter ablation with $97 \%$ sensitivity and $100 \%$ specificity $^{(1-3)}$. Intracardiac echocardiography (ICE) has also been proven to provide a similar real time high-resolution imaging capability, which makes it an ideal technique during LA transeptal ablation procedures $^{(4)}$. On the other hand computed tomography $(\mathrm{CT})$ and cardiac magnetic resonance (CMR) are commonly utilized to plan procedures by visualizing pulmonary veins and LA anatomy, and there is growing evidence suggesting that these advanced imaging techniques can provide a non-invasive alternative to TEE for evaluation of LA thrombus ${ }^{(5,6)}$. Guidelines are limited on the precise role of advanced imaging techniques for thrombus evaluation prior to LA ablation ${ }^{(7)}$.

We analyzed LA findings among patients with AF undergoing a CT/CMR study for preprocedure evaluation for PVI, and compared them to the intraprocedural ICE evaluation 
of the LA. Our hypothesis was whether there was correlation between the findings of the CT/CMR and the intraprocedural ICE. The aim of our study was to correlate the CT/MR and intraprocedural ICE findings and determine whether CT/CMR could reduce the need for TEE and/or ICE prior to or during catheter ablation of AF.

\section{Methods}

\section{Population}

Consecutive patients undergoing PVI for recurrent from 2012 to 2017 and had a preprocedural CT/CMR were retrospectively included. Only patients with anticoagulation started at least 3 weeks before performance of the CT/CMR and uninterrupted until the ablation procedure were selected. In all patients an ICE catheter was used to rule out the presence of LA thrombus before the transeptal puncture, and also for transeptal puncture guidance. The study was conducted in compliance with the most recent version of the Declaration of Helsinki, Spanish laws andregulations (Royal Decree 1090/2015, Royal Decree 1616/2009, Order SAS/3470/2009 of 16December). The study was assessed and approved by the Ethical Committee of Hospital de Mar,Comité Ético de Investigación Clínica del Consorci Mar Parc de Salut de Barcelona (CEIC-Parc DeSalut Mar). All patients signed informed consent before inclusion in the registry.

\section{Imaging protocols}

CT Imaging protocol. Multi-slice CT and retrospectively ECG-gated spiral acquisitions were performed. In order to improve the specificity a two-phase scan protocol was used, acquiring a 
second set of images with a short delay after the initial scan. Traditionally the images are captured only few seconds after arrival of contrast to the left heart, making it difficult to differentiate thrombus from sluggish flow. The addition of delayed imaging allows a better distinction between these two elements, considering that a filling defect that persists 1 minute after contrast injection is more likely to represent thrombus, whereas sluggish flow is more likely to show contrast opacification during delayed images ${ }^{(6)}$. ECG gating improves imaging quality (see Figure 1).

CMR Imaging protocol. A 1.5 Tesla GE system (GE Signa Excite, GE Healthcare, Ireland) coupled to a 6-element body phased array coils were used. Transverse, coronal and sagittal plane localizing images were acquired using a FIESTA sequence. Cine-CMRI in the 4 and 2chamber orientations using steady-state free precession pulse sequences in held end expiration was used. Temporal resolution of cine steady-state free precession images was 30 to $40 \mathrm{~ms}$ adjusted according to the patient's heart rate and ability to breathe hold. Late gadolinium enhancement images were obtained after intravenous injection of $0.2 \mathrm{mmol} / \mathrm{kg}$ of a gadolinium chelate contrast agent (Dotarem, Gd-DOTA, Guerbet, Villepinte, France).

ICE Imaging protocol. The test was performed using a Sequoia ultrasound system with a ViewFlex Xtra ICE catheter (Abbott). During each procedure, the ICE catheter was inserted into the left femoral vein through a 10F introducer sheath and advanced into the right atrium (RA). The ICE catheter manipulation included rotation, advancement, withdrawal and 
possibility of antero-posterior and right to left deflections through two jog dials. Once in the RA, by gradual clockwise rotation of the catheter we can obtain panoramic views of the interatrial septum, LA, mitral annulus, LA appendage, and PVs. ICE was used to rule out the presence of thrombus in any chamber, including the LA appendage (see Figure 2). Subsequently it was used to identify the fossa ovalis to guide the transeptal puncture and help in positioning the ablation catheter in the pulmonary vein antrum. In our center approximately 100 transseptal punctures are performed yearly since 2009, always under the ICE guidance. Thus, operators have developed high cognitive and technical skills concerning this advanced technique.

\section{Statistical analysis}

Continuous variables were presented as mean \pm standard deviation or median. The categorical data were expressed as frequencies and percentages. Univariate comparisons were performed using the $\chi 2$ test for categorical variables. A p value of $<0.05$ was considered statistically significant. All analyses were performed using the SPSS for Mac version 20.0 (SPSS Inc., Chicago, IL, United States).

\section{Results}

\section{Population characteristics}

We included 272 consecutive patients averaging $54.5 \pm 4.94$ years (71\% male). Mean AF evolution time was 3.3 years. The population was relatively healthy with a CHA2DS2VASC 
below 2 in $72 \%$ and a LA diameter of $42 \pm 5.7 \mathrm{~mm}$. Up to $22 \%$ of patients had an anatomic variation of the PV, mainly a left common ostium (see Table 1).

\section{Anticoagulation therapy}

Of the 272 patients, 111 were on Acenocumarol (40\%), and 161 patients were on direct oral anticoagulants: 38 patients were on Dabigatran (14\%), 55 on Rivaroxaban (20), 63 on Apixaban

(23\%), and 5 on Edoxaban (1\%). Anticoagulation was started $227 \pm 392$ days before the performance of CT/CMR, and $291 \pm 416$ days before the ablation procedure (see Table 2 ) and it was not discontinued during the ablation. Mean basal ACT, just before the daily anticoagulation

dose given prior to the procedure was $162.2 \pm 32.6$, and mean ACT during the procedure was $310.4 \pm 73.4$

\section{Thrombus detection}

CT/CMR was diagnostic of intracardiac thrombus in only 2 out of the 272 cases $(0.7 \%$; both within the LA appendage). Those two patients, which were on acenocumarol with a TTR of $70 \%$, remained on anticoagulation for an additional 6 months period with a higher INR goal and increased INR controls, after which CT/CMR was negative for intracardiac thrombus. 
Time between the CT/CMR and the procedure was $64 \pm 142$ days. At the time of the procedure no macroscopic thrombus was observed in any patients with ICE. Therefore CT/CMR achieved a negative predictive value of $100 \%$ compared to ICE $(\mathrm{p}<0.01)$.

\section{Costs analyses}

From an economic point of view in the setting a public health system the cost of the use of CT or CMR previous to AF ablation procedures to rule out intracardiac thrombus needs to be weighed against the cost of use of TEE or ICE. The cost of a CT scan or a CMR in our hospital is approximately 250 euros, while only the cost of an ICE catheter is 1900 euros. Regarding the TEE, CT/CMR has the advantages of being noninvasive, with shorter procedural time, and without the need for anesthesia support, as well as providing left atrial and pulmonary vein structure, which is useful for procedural planning.

\section{Follow-up}

Only one patient experienced a catheter ablation related stroke (0.3\%), defined as occurring within the first month after the procedure. This patient received appropriate oral anticoagulation therapy (acenocumarol) before the procedure, with a TTR of $80 \%$ and INR controls every 4 weeks, starting 1537 days before the ablation, and uninterrupted after the procedure, since his CHA2DSVAS2C score was 3. The ACT for this patient had been $>250$ sec during all the procedure, which had been particularly long and complex due to difficulties to isolate the left superior PV. No thrombus was observed either with a CT scan performed 32 days before the ablation, nor with the periprocedural ICE. 


\section{Discussion}

Catheter ablation has been established as a standard treatment for symptomatic AF and PVI has become the cornerstone of such treatment, as recommended in the current guidelines ${ }^{(8)}$. Several studies have shown that advanced cardiac imaging techniques can improve AF ablation result ${ }^{(9,10)}$. Both CT scan and CMR provide accurate anatomic details (i.e. number and size of the PVs and the LA, and anatomic variants of the PVs) in a non-invasive manner, and can help the electrophysiologist to plan the procedure. Furthemore advanced image techniques can be integrated to the $3 \mathrm{D}$ navigation systems used to perform $\mathrm{AF}$ ablation procedures which can provide real-time non-fluoroscopic intraprocedural navigation guidance ${ }^{(11-13)}$. Patients with $\mathrm{AF}$ have increased risk of stroke from left atrial thrombi. Consequently, the need to rule out intracardiac thrombus is mandatory prior to the ablation procedure. Historically, TEE has been considered the "gold standard" imaging technique in order to rule out left atrial thrombus, and it is usually performed within 24 to 48 hours before the procedure. However TEE is an invasive procedure that requires sedation and sometimes intubation. Therefore other alternatives have been inverstigated: ICE can both rule out the presence of thrombus and guide the procedure ${ }^{(14,15)}$, but at a high cost. On the other hand other advanced imaging techniques, such CT scan or CMR have been increasingly used in current clinical practice, and may be able to replace TEE or ICE in a non-invasive manner. Several studies investigated the use of CT scan and CMR for the assessment of LA thrombus in pre-procedural evaluation for AF ablation. A metanalysis from Romero et al. showed an overall high accuracy of CT scan compared to TEE for the detection of LA/LAA thrombus. Other metanalysis of prospective studies comparing CT with TEE have had similar results and support the use of CT to rule out LA thrombus, especially when delayed imaging, ECG gating and heart rate control are 
performed ${ }^{(16-17)}$. Similar evidence has been demonstrated for CMR as well, which is another noninvasive technique that has been shown to be as effective as TEE in evaluating for LA thrombus, in addition to providing LA and PV anatomy and structure ${ }^{(18-21)}$. None of these studies compared advanced imaging techniques versus ICE. The purpose of our study was to examinate the cost-effective and time-efficient advantatges of pre-procedural CT scan or CMR performed remotely (in our case an average of two months pre-procedure) compared to the use of TEE or ICE, to evaluate for LA thrombus prior to AF ablation. We found this strategy to be a safe, non-invasive and cost-effective alternative for LA thrombus detection. Importantly this was true even for deferred procedures as long as the anticoagulation therapy continued uninterrupted. To our knowledge, this is the largest study comparing the accuracy of LA thrombus detection with non-invasive advanced image techniques compared to periprocedural ICE.

In conclusion it seems clear that employing pre-procedural CT/CMR as the only strategy to rule- out LA/LAA thrombus is efficient and safe as well as being non-invasive. It also appears, as previously described, that the use of CT/CMR can limit procedural costs while preserving high-quality care ${ }^{(22,23)}$.

\section{CONCLUSION}

CT and CMR are an excellent surrogate of TEE or ICE to rule out intracardiac thrombus in patients adequately anticoagulated prior to AF ablation. This is true even for deferred procedures as long as anticoagulation is uninterrupted. Furthemore the pre-procedural imaging provides key anatomic information that can be complemented by intraprocedural electroanatomic mapping to minimize procedural complications. 


\section{References:}

1. Manning WJ, Weintraub RM, Waksmonski CA, Haering JM, Rooney PS, Maslow AD et al. Accuracy of transesophageal echocardiography for identifying left atrial thrombi. A prospective, intraoperative study. Ann Intern Med 1995;123:817-22. DOI: 10.7326/0003-4819123-11- 199512010-00001.

2. Hahn RT, Abraham T, Adams MS, Bruce CJ, Glas KE, Lang RM et al. Guidelines for performing a comprehensive transesophageal echocardiographic examination: recommendations from the American Society of Echocardiography and the Society of Cardiovascular Anesthesiologists. J Am Soc Echocardiogr. 2013;26:921-64. DOI: 10.1016/j.echo.2013.07.009.

3. Hilberath JN, Oakes DA, Shernan SK, Bulwer BE, D’Ambra MN, Eltzschig HK. Safety of transesophageal echocardiography. J Am Soc Echocardiogr. 2010;23:1115-27. DOI: 10.1016/j.echo.2010.08.013.

4. Sriram CS, Banchs JE, Moukabary T, Moradkhan R, Gonzalez MD. Detection of left atrial thrombus by intracardiac echocardiography in patients undergoing ablation of atrial fibrillation. J Interv Card Electrophysiol. 2015 Sep;43:227-36. DOI: 10.1007/s10840-015-0008-2. 
5. Choi YRKH-L, Kwon H-M, Chun EJ, Ko SM, Yoo SM, Choi S-I et al. Cardiac CT and MRI for assessment of cardioembolic stroke. Cardiovasc Imaging Asia 2017;1:13-22. https://doi.org/10.22468/cvia.2016.00045

6. Romero J, Husain SA, Kelesidis I, Sanz J, Medina HM, Garcia MJ. Detection of left atrial appendage thrombus by cardiac computed tomography in patients with atrial fibrillation: a meta- analysis. Circ Cardiovasc Imaging 2013;6:185-94. DOI:

10.1161/CIRCIMAGING.112.000153.

7. Calkins H, Hindricks G, Cappato R, Kim YH, Saad EB, Aguinaga L, et al. 2017 HRS/EHRA/ECAS/APHRS/SOLAECE expert consensus statement on catheter and surgical ablation of atrial fibrillation. Europace. 2018; 20(1): e1-e160. DOI: 10.1093/europace/eux275.

8. Hindricks G, Potpara TS, et al. 2020 ESC Guidelines for the diagnosis and management of atrial fibrillation developed in collaboration with the European Association of Cardio-Thoracic Surgery (EACTS). Eur Heart J.2020. DOI: 10.1093/eurheartj/ehaa612

9. Obeng-Gyimah E, Nazarian S. Advancements in Imaging for Atrial Fibrillation Ablation: Is There a Potential to Improve Procedural Outcomes? J Innov Card Rhythm Manag. 2020;11:4172-4178. DOI: 10.19102/icrm.2020.110701

10. Rathi VK, Reddy ST, Anreddy S, et al. Contrast-enhanced CMR is equally effective as TEE in the evaluation of left atrial appendage thrombus in patients with atrial fibrillation undergoing 
pulmonary vein isolation procedure. Heart Rhythm. 2013;10:1021-1027. DOI: 10.1016/j.hrthm.2013.02.029.

11. Tops LF, Bax JJ, Zeppenfeld K, Jongbloed MR, Lamb HJ et al. Fusion of multislice computed tomography imaging with three-dimensional electroanatomic mapping to guide radiofrequency catheter ablation procedures. Heart Rhythm. 2005;2:1076-1081. DOI: 10.1016/j.hrthm.2005.07.019.

12. Kistler PM, Rajappan K, Jahngir M, Earley MJ, Harris S, et al. The impact of CT image integration into an electroanatomic mapping system on clinical outcomes of catheter ablation of atrial fibrillation. J Cardiovasc Electrophysiol. 2006;17:1093-1101. DOI: 10.1111/j.15408167.2006.00594.

13. Hamdan A, Charalampos K, Roettgen R, et al. Magnetic resonance imaging versus computed tomography for characterization of pulmonary vein morphology before radiofrequency catheter ablation of atrial fibrillation. Am $\mathrm{J}$ Cardiol. 2009;104:1540-154. DOI: 10.1016/j.amjcard.2009.07.029. 
14. Saucedo J, Martinho S, Frankel D,et al. Exclusion of Left Atrial Appendage Thrombus Using Single Phase Coronary Computed Tomography as Compared to Transesophageal Echocardiography in Patients Undergoing Pulmonary Vein Isolation. International Scholarly Research Notices. 2014,4:838727. https://doi.org/10.1155/2014/838727.

15. Hur J, Pak HN, Kim YJ, et al. Dual-enhancement cardiac computed tomography for assessing left atrial thrombus and pulmonary veins before radiofrequency catheter ablation for atrial fibrillation. Am J Cardiol. 2013;112:238-244. DOI: 10.1016/j.amjcard.2013.03.018.

16. Romero J, Husain S., Kelesidis I, et al. Detection of left atrial appendage thrombus by cardiac computed tomography in patients with atrial fibrillation: a meta-analysis. Circulation: Cardiovascular Imaging, 2013; 6:185-194. https://doi.org/10.1161/CIRCIMAGING.112.000153.

17. Zou H, Zhang Y, Tong J, Liu Z. Multidetector computed tomography for detecting left atrial/left atrial appendage thrombus: a meta-analysis. Intern Med J. 2015;45:1044-1053. DOI: 10.1111/imj.12862.

18. Caponi D, Corleto A, Scaglione M, et al. Ablation of atrial fibrillation: does the addition of three-dimensional magnetic resonance imaging of the left atrium to electroanatomic mapping improve the clinical outcome? A randomized comparison of Carto-Merge vs. Carto-XP threedimensional mapping ablation in patients with paroxysmal and persistent atrial 
fibrillation. Europace. 2010;12:1098-1104. DOI: 10.1093/europace/euq107. DOI: 10.1093/europace/euq107.

19. Rathi VK, Reddy ST, Anreddy S, et al. Contrast-enhanced CMR is equally effective as TEE in the evaluation of left atrial appendage thrombus in patients with atrial fibrillation undergoing pulmonary vein isolation procedure. Heart Rhythm. 2013;10:1021-1027. DOI: 10.1016/j.hrthm.2013.02.029.

20. Blomstrom Lundqvist C, Auricchio A, Brugada J, et al. The use of imaging for electrophysiological and devices procedures: a report from the first European Heart Rhythm Association Policy Conference, jointly organized with the European Association of Cardiovascular Imaging (EACVI), the Council of Cardiovascular Imaging and the European Society of Cardiac Radiology. Europace. 2013;15:927-936. DOI: 10.1093/europace/eut084.

21. Baran J, Stec S, Pilichowska-Paszkiet E, et al. PIntracardiac echocardiography for detection of thrombus in the left atrial appendage: comparison with transesophageal echocardiography in patients undergoing ablation for atrial fibrillation: the Action-Ice I Study. Circ Arrhythm Electrophysiol. 2013 Dec;6(6):1074-81. DOI: 10.1161/CIRCEP.113.000504.

22. Use of Intracardiac Echocardiography in Interventional Cardiology Working With the Anatomy Rather Than Fighting It Andres Enriquez, Luis C. Saenz, Raphael Rosso , Frank E. Silvestry, David Callans, Francis E. Marchlinski, and Fermin Garcia. DOI: 10.1161/circulationaha.117.031343. 
23. Pokorney SD, Hammill BG, Qualls LG, Steinberg BA, Curtis LH, Piccini JP. Cost analysis of periprocedural imaging in patients undergoing catheter ablation for atrial fibrillation. Am J Cardiol. 2014 Jul 15;114(2):266-71. doi: 10.1016/j.amjcard.2014.04.031. Epub 2014 May 2. PMID: 24952929; PMCID: PMC4090097. DOI: 10.1016/j.amjcard.2014.04.031.

\section{Figure legends}

Figure 1: CT scan caption showing the absence of thrombus in the LA, including the LAA. LA: left atrium; LAA: left atrium appendage; LV: left ventricle; MA: mitral annulus.

Figure 2: ICE caption with the transducer placed into de low right atrium, where absence of thrombus can be observed in the LA, including the LAA. LA: left atrium; LAA: left atrium appendage; LV: left ventricle; MA: mitral annulus; RA: right atrium. 


\section{Tables}

Table 1. Population characteristics

\begin{tabular}{|l|c|}
\hline & Patients=272 \\
\hline Age: years (ave \pm st dev) & $54.5 \pm 4,94$ \\
\hline Gender, male: $\mathrm{n}(\%)$ & $194(71)$ \\
\hline Hypertension: $\mathrm{n}(\%)$ & $109(40)$ \\
\hline
\end{tabular}

\begin{tabular}{|l|c|}
\hline CHA2DS2VASC score <2 & $197(72)$ \\
\hline Type, persistent AF: $\mathrm{n}(\%)$ & $38(14)$ \\
\hline AF evolution time: months (ave \pm st dev) & $40 \pm 41.4$ \\
\hline LV ejection fraction: (ave \pm st dev) & $59 \pm 9.3$ \\
\hline LA diameter: mm (ave \pm st dev) & $42 \pm 5.7$ \\
\hline Image tecnique: $\mathrm{n}(\%)$ & $147(54)$ \\
CT scan & $125(46)$ \\
CMR & $55(20)$ \\
\hline PV anatomic variation: $\mathrm{n}(\%)$ & \\
\hline
\end{tabular}

AF: atrial fibrillation; Ave: average; CMR: cardiac magnetic resonance; CT: computed tomography; LA: left atrium; LV: left ventricle; PV: pulmonary veins; st dev: standard deviation 
Table 2. Treatment and procedural characteristics

\begin{tabular}{|l|c|}
\hline & Total patients=272 \\
\hline Time from AC to ablation: days (ave \pm st dev) & $227 \pm 392$ \\
\hline Time from CT/CMR to ablation: days (ave \pm st dev) & $291 \pm 416$ \\
\hline Type of ablation: $\mathrm{n}(\%)$ & $89(33)$ \\
RF ablation & $183(67)$ \\
Cryoballoon ablation & \\
\hline Anticoagulant drugs: $\mathrm{n}(\%)$ & $38(14)$ \\
\hline Dabigratan & $55(20)$ \\
\hline Rivaroxaban & $63(23)$ \\
\hline Apixaban & $5(1)$ \\
\hline Edoxaban & $111(40)$ \\
\hline Acenocumarol & $162.2 \pm 32.6$ \\
\hline Basal ACT: seconds (ave \pm st dev) & $310.4 \pm 73.4$ \\
\hline 30 minutes ACT: seconds (ave \pm st dev) & \\
\hline
\end{tabular}

AC: anticoagulation; ACT: activated clotting time; Ave: average; CMR: cardiac magnetic resonance; CT: computed tomography; RF: radiofrequency; st dev: standard deviation 


\section{Figures}

Figure 1. CT

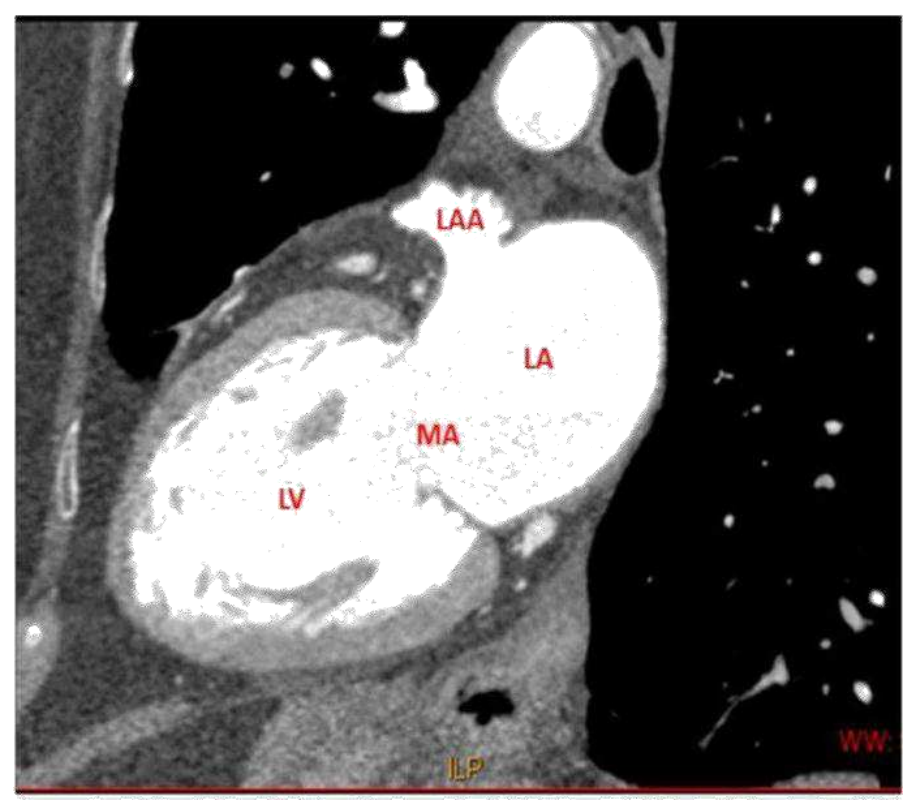

Figure 2. ICE

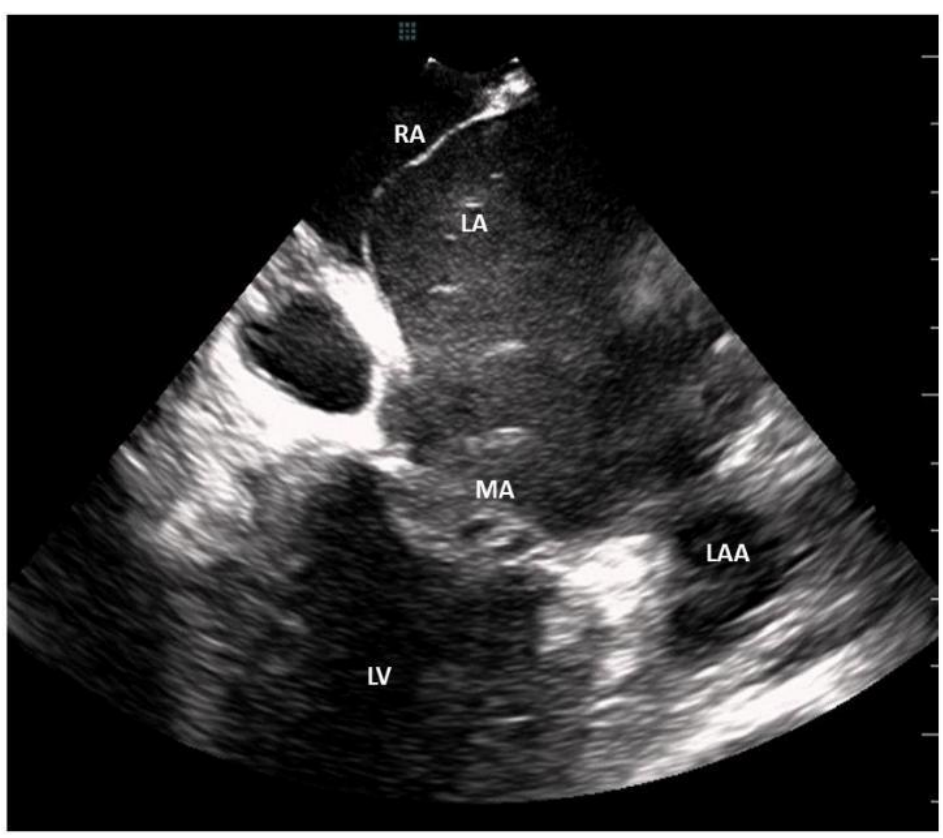

